\title{
Proteomic analysis of the soluble proteomes of miltefosine-sensitive and -resistant Leishmania infantum chagasi isolates obtained from Brazilian patients with different treatment outcomes
}

\author{
Juliana B.T. Carniellia,b, Hélida M. de Andrade ${ }^{c}$, Simone F. Pires ${ }^{c}$, \\ Alexander D. Chapeaurouge ${ }^{d}$, Jonas Perales ${ }^{d}$, Renata Monti-Rocha ${ }^{a}$, Sílvio F.G. Carvalho ${ }^{e}$, \\ Leonardo P. Ribeiro ${ }^{b}$, Reynaldo Dietze ${ }^{a}$, Suely G. Figueiredo ${ }^{b}$, Elenice M. Lemos ${ }^{a, *}$ \\ ${ }^{a}$ Laboratório de Leishmanioses, Núcleo de Doenças Infecciosas, Universidade Federal do Espírito Santo, Vitória, ES, Brazil \\ bLaboratório de Química de Proteínas, Departamento de Ciências Fisiológicas, Universidade Federal do Espírito Santo, Vitória, ES, Brazil \\ ${ }^{\mathrm{C}}$ Laboratório de Leishmanioses, Departamento de Parasitologia, Universidade Federal de Minas Gerais, Belo Horizonte, MG, Brazil \\ dLaboratório de Toxinologia, Instituto Oswaldo Cruz, Fiocruz, Rio de Janeiro, RJ, Brazil

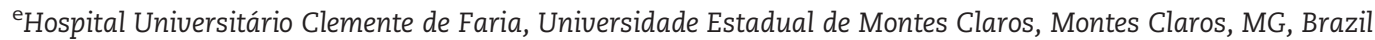

\section{A R T I C L E I N F O}

\section{Article history:}

Received 14 February 2014

Accepted 17 May 2014

Available online 27 May 2014

\section{Keywords:}

L. infantum chagasi

Miltefosine resistance

Proteome

2D-DIGE

MS/MS

\begin{abstract}
A B S T R A C T
The mechanism of miltefosine-resistance in Leishmania spp. has been partially determined in experimental resistant lines; however, studies using clinical isolates with different miltefosine susceptibilities are still needed. In our study, we used a proteomic 2D-DIGE/MS approach to study different protein abundances in miltefosine-sensitive and -resistant Leishmania infantum chagasi isolates from visceral leishmaniasis patients with different miltefosine treatment outcomes. The high-resolution proteome obtained from these isolates showed 823 matched spots and 46 spots exhibited different abundances between the isolates. Out of these differentially expressed spots, 26 (56.5\%) showed greater and 20 (43.5\%) showed lower expression of the resistant isolate compared to the sensitive isolate. MALDI/TOF-TOF mass spectrometry allowed the identification of 32 spots with unique protein identification correspondent to 22 non-redundant proteins. Most of the proteins up-regulated in the proteome miltefosine-resistant isolates were associated with redox homeostasis, stress response, protection to apoptosis, and drug translocation. These differentially expressed proteins are likely involved in miltefosine natural resistance and suggest that the miltefosine-resistance mechanism in Leishmania is multifactorial.
\end{abstract}

Biological significance

Visceral leishmaniasis (VL) is a serious disease with a challenging treatment plan requiring the prolonged and painful applications of poorly tolerated toxic drugs. Therefore, the

Abbreviations: VL, Visceral leishmaniasis; R, Miltefosine-resistant isolate; S, Miltefosine-sensitive isolate; 2D-DIGE, Two Dimensional Difference Gel Electrophoresis.

* Corresponding author. Tel.: +55 273335 7208; fax: +55 2733357206

E-mail address: lemosem@ndi.ufes.br (E.M. Lemos). 
identification of miltefosine, an effective and safe oral drug, was considered a significant advancement in leishmaniasis therapy. However, different sensitivities to miltefosine in Leishmania have been observed in clinically relevant species, and the biological mechanism by which clinical isolates of Leishmania acquire drug resistance is poorly understood. Our work aims to elucidate the mechanism of natural resistance to miltefosine in Leishmania by studying the isolates from VL patients who displayed different miltefosine treatment outcomes.

(c) 2014 Elsevier B.V. All rights reserved.

\section{Introduction}

American visceral leishmaniasis, caused by Leishmania infantum chagasi, is a major health problem in many parts of Brazil. The disease is characterised clinically by fever, gradual weight loss, splenomegaly, hypergammaglobulinemia, and pancytopenia and is usually fatal without specific treatment [1].

One of the most significant advances in leishmaniasis therapy was the identification of an effective and safe oral drug, miltefosine (hexadecylphosphocholine), a phospholipid analogue. Miltefosine was originally developed as an anticancer drug and is the first oral drug approved for visceral leishmaniasis (VL) in India [2,3].

While miltefosine was able to produce a clinical cure in approximately $90 \%$ of VL caused by L. donovani [4,5], a clinical trial showed that the cure rate in Brazil, where VL is caused by $\mathrm{L}$. infantum chagasi, was much lower. This study revealed that the cure rate was only $43 \%$ with a regimen of $2.5 \mathrm{mg} / \mathrm{kg}$ daily for 28 days and $67 \%$ with an extended treatment of 42 days with the same dose. Pre-treatment isolates obtained from cured patients and relapsed patients, were susceptible and resistant in vitro, respectively, suggesting that therapeutic failure may be due to intrinsic parasitic resistance to miltefosine (Dietze, R., unpublished data).

Miltefosine resistance mechanisms in Leishmania spp. have been associated with a decrease in intracellular drug accumulation due to the defective inward translocation of miltefosine [6-9] and the over-expression of ABC transporters, which are responsible for drug efflux [10]. Studies have shown that alterations in fatty acid and sterol biosynthesis result in a change in the lipid composition of membranes in miltefosineresistant $\mathrm{L}$. donovani, which could alter membrane fluidity and permeability and affect the drug-membrane interaction [11]. In addition, low susceptibility to drug-mediated programmed cell death has been observed in miltefosine-resistant Leishmania [12,13].

However, considering that the mechanism of miltefosine resistance in Leishmania spp. has been partially understood in experimental resistant lines, studies using clinical isolates with different miltefosine susceptibility are still needed. Taking into account the fact that proteins are ultimately responsible for cell phenotypes, in the present study we performed a comparative proteomics screen between miltefosine-sensitive and -resistant Brazilian L. infantum chagasi clinical isolates from VL patients with different miltefosine treatment outcomes. Using this approach, we identified differentially expressed proteins that may link molecular components to pathways involved in natural miltefosine resistance in Leishmania.

\section{Materials and methods}

\subsection{Ethics statement}

Written consent was obtained from patients or from their parents or guardians for children. Ethical clearance was obtained from the institutional review board of the Centro de Ciências da Saúde, Universidade Federal do Espírito Santo (CEP-066/2007), Brazil. The animal experiments were approved by the Committee on the Ethics of Animal Experiments of the Centro de Ciências da Saúde, Universidade Federal do Espírito Santo (CEP-009/2010), Brazil, and all efforts were made to minimise suffering.

\subsection{Patients and parasites}

We studied a pair of L. infantum chagasi isolates, one from a cured patient (MHOM/BR/05/MG14) and the other from a patient who relapsed after miltefosine treatment (MHOM/BR/05/MG11). These isolates were obtained from bone marrow aspirates taken before treatment of VL patients, participants in a clinical trial (Minas Gerais state, Brazil) to evaluate the efficacy and toxicity of miltefosine. The patients were treated with $2.5 \mathrm{mg} / \mathrm{kg} /$ day of miltefosine for 28 days and were followed for a minimum of six months after treatment was terminated. Patients were considered cured if no signs and symptoms of the disease were present at the time of examination. Relapse was defined as a patient who was considered cured, but upon follow-up, showed reappearance of clinical signs and positive parasitology. The clinical isolates selected for this study were identified as L. infantum chagasi based on a PCR-RFLP assay [14].

\subsection{Parasite culture}

Promastigotes were grown in liver infusion tryptose (LIT) medium supplemented with $10 \%$ heat-inactivated foetal calf serum $\mathrm{pH} 7.5,25 \stackrel{\circ}{\circ} \mathrm{C}$. The cultures were initiated by inoculating parasites into culture medium to a final concentration of $10^{6}$ parasites $/ \mathrm{mL}$. Cell number was determined microscopically using a Neubauer chamber. Three independently growing cultures of each isolate ( $\mathrm{S}$ and $\mathrm{R}$ ) were obtained and were further pooled.

\subsection{Susceptibility of intracellular amastigotes to miltefosine}

Late log stage promastigotes were used to infect primary peritoneal macrophages isolated from a Swiss mouse at a ratio of 7 promastigotes to 1 macrophage. The cells were plated in RPMI 1640 medium plus 10\% heat-inactivated foetal calf serum in 16-well Lab-Tek ${ }^{\mathrm{TM}}$ tissue culture chamber slides (Scientific Laboratory Supplies, Wilford, UK) [15] and maintained overnight 
at $37{ }^{\circ} \mathrm{C}$ in a $5 \% \mathrm{CO}_{2} / 95 \%$ air mixture. Extracellular parasites were removed by washing, and fresh medium containing miltefosine was added at a final concentration ranging from 0.55 to $15 \mu \mathrm{M}$. Higher concentrations were not tested due to toxicity to infected macrophages. Each concentration was tested in triplicate. After $72 \mathrm{~h}$ of incubation at $37{ }^{\circ} \mathrm{C}$ in a $5 \% \mathrm{CO}_{2} / 95 \%$ air mixture, the cultures were methanol-fixed and Giemsa-stained and 100 macrophages/well were microscopically evaluated. Drug activity was determined from the percentage of infected cells in drug-treated cultures relative to non-treated cultures. The mean effective concentration $\left(\mathrm{EC}_{50}\right)$ values were calculated by nonlinear regression analysis using Prism version $5.0 \mathrm{~b}$ software. The results are expressed as the means and standard deviation of three independent experiments.

\subsection{Protein extract}

The protein extracts were obtained simultaneously for all samples. Promastigotes were obtained in the late log phase in LIT medium. Cells were harvested by centrifugation at $1000 \mathrm{~g}$ for 10 min at $4{ }^{\circ} \mathrm{C}$, washed three times in RPMI 1640 medium, and resuspended in two-dimensional lysis buffer ( $8 \mathrm{M}$ urea, $2 \mathrm{M}$ thiourea, $4 \%$ CHAPS, $40 \mathrm{mM}$ Tris base and a cocktail of protease inhibitors (Sigma-Aldrich)) in a proportion of $250 \mu \mathrm{L}$ of lysis buffer for every $10^{9}$ parasites. Lysis was allowed to proceed for $2 \mathrm{~h}$ by gently vortexing at room temperature, followed by passing the homogenate through a 26-gauge needle 10 times. Then, the samples were centrifuged at $20,000 \mathrm{~g}$ for $30 \mathrm{~min}$ to remove insoluble material. Protein concentration was determined using the 2D Quant Kit (GE Healthcare). Protein extracts were aliquoted into single-use sample and stored at $-80^{\circ} \mathrm{C}$ until analysis.

\subsection{Two-dimensional difference gel electrophoresis (2D-DIGE)}

Previously three representative Coomassie 2D gels from three independent biological replicates of each isolate ( $S$ and $R$ ) were analysed and the profiles obtained were highly reproducible regarding protein spot total number, relative positions and intensities (data not shown). Considering these data, in our DIGE analyse was used a pool of three biological replicate of each isolate ( $S$ and R). A total of 3 2D-DIGE were performed, thus three different images of each pool of isolates were obtained.

The $\mathrm{pH}$ of the protein extracts was checked and adjusted to 8.5 with $50 \mathrm{mM} \mathrm{NaOH}$, if necessary. Each fifty-microgram sample was labelled with 400 pmol of N-hydroxysuccinimidyl-ester derivate of cyanine dyes Cy2, Cy3, and Cy5 according to the manufacturer's instructions for minimal labelling dyes (GE Healthcare). Briefly, samples from miltefosine-sensitive and -resistant isolates were labelled with Cy3 or Cy5. To minimise the potential confounding effects of differences in fluorescence intensity, Cy3 and Cy5, labelling was alternated between sensitive and resistant pool extracts in a 'balanced' design. An internal standard, which comprised a mixture of equal amounts of both isolates, was labelled with Cy2. Labelling was performed for $30 \mathrm{~min}$ on ice in the dark. The reactions were then quenched by the addition of $1 \mu \mathrm{L}$ of lysine $(10 \mathrm{mM})$ for $10 \mathrm{~min}$ on ice in the dark. Labelled samples were combined, in such a way that each mixture comprised protein samples from miltefosine-sensitive and -resistant isolates and an aliquot of the internal standard. Then, the total volume was adjusted to $350 \mu \mathrm{L}$ with Destreak rehydration solution (GE Healthcare) supplemented with $0.2 \%$ ampholytes 4-7 (Bio-Rad).

Samples were applied to the immobilised $\mathrm{pH}$ gradient (IPG) strips, $18 \mathrm{~cm} \mathrm{pH} \mathrm{4-7} \mathrm{(GE} \mathrm{Healthcare),} \mathrm{by} \mathrm{in-gel} \mathrm{sample} \mathrm{rehydra-}$ tion, and passive rehydration was performed for $20 \mathrm{~h}$ in the dark. After rehydration, isoelectric focusing was performed on an IPGphor II (GE Healthcare) at a temperature of $20{ }^{\circ} \mathrm{C}$ and a maximum current of $50 \mathrm{~mA} /$ strip. The focusing parameters were as follows: step 1-300 V constant for 5 h; step 2-gradient from 300 to $10,000 \mathrm{~V}$ over $2 \mathrm{~h}$; step 3-10,000 V until complete $60,000 \mathrm{Vh}$; and step 4-500 V constant for $5 \mathrm{~h}$. After isoelectric focusing, each strip was incubated for $15 \mathrm{~min}$ in equilibration buffer (50 mM Tris-HCl, pH 8.8, $6 \mathrm{M}$ urea, 30\% glycerol, 2\% SDS, trace of bromophenol blue) containing $10 \mathrm{mg} / \mathrm{mL}$ dithiothreitol, followed by a second incubation in equilibration buffer containing $25 \mathrm{mg} / \mathrm{mL}$ iodoacetamide for $15 \mathrm{~min}$. IPG strips were sealed to $12 \%$ acrylamide gels using $0.5 \%$ agarose in standard Tris-Glycine-SDS electrophoresis buffer. The second dimension SDS-PAGE was carried out on the Ettan DaltSix system at $16 \mathrm{~mA} /$ gel for $30 \mathrm{~min}$ and then at $40 \mathrm{~mA} / \mathrm{gel}$ until the dye front reached the bottom of the gel at $15{ }^{\circ} \mathrm{C}$.

Two-dimensional gels cast between two low-fluorescence glass plates were scanned with the Typhoon FLA 9000 (GE Healthcare) at a resolution of $100 \mu \mathrm{m}$, and photomultiplier (PTM) values were adjusted to optimise sensitivity and prevent oversaturation. The excitation/emission wavelengths for Cy2, Сy3, and Cy5 were 488/520, 532/580, and 633/670, respectively. Following fluorescence scanning, DIGE gels were also stained with colloidal Coomassie CBB G-250, following procedures described elsewhere [16], to allow the visual detection of differential abundances of spots.

\section{7. $2 \mathrm{D}$ image analysis}

Analysis of 2D-DIGE was done using DeCyder 2D software, version 7.0 (GEHealthcare) according to the manufacturer's recommendations. The t-test with false discovery rate correction was used for the statistical analysis of the data, and $\alpha<0.05$ was adopted as the level of significance. Protein spots that showed differential abundance in sensitive and resistant L. infantum chagasi clinical isolates were selected for mass spectrometry identification.

\subsection{Protein identification}

Spots with differential expression were excised, and gel fragments were washed twice with 50\% acetonitrile in $25 \mathrm{mM}$ ammonium bicarbonate, followed by pure acetonitrile. The gel fragments were dried and then rehydrated with $10 \mu \mathrm{L}$ of protease solution (Trypsin Gold, Mass Spectrometry Grade, Promega, at $20 \mathrm{ng} / \mu \mathrm{L}$ in $50 \mathrm{mM}$ ammonium bicarbonate) for $30 \mathrm{~min}$ on ice. Then, $20 \mu \mathrm{L}$ of $50 \mathrm{mM}$ ammonium bicarbonate was added, and digestion was performed at $37{ }^{\circ} \mathrm{C}$ for $16 \mathrm{~h}$. Peptide extraction was performed twice for 30 min with $30 \mu \mathrm{L}$ of $50 \%$ acetonitrile/5\% formic acid. Trypsin digests were then concentrated in a SpeedVac (Eppendorf) to about $10 \mu \mathrm{L}$ and desalted using Zip-Tip (C18 resin; P10, Millipore). Peptide elution from the column was performed in $50 \%$ acetonitrile/ 
$0.1 \%$ trifluoroacetic acid [17]. A gel fragment with no protein and a gel fragment from the molecular weight standard bovine albumin were used as negative and positive controls, respectively.

The tryptic peptides were analysed with a MALDI-TOF-TOF AB Sciex 5800 (AB Sciex, Foster City, CA) mass spectrometer. MS and MSMS spectra were acquired in reflector mode to ensure optimal mass accuracy and peak resolution. Usually up to 15 of the most intense ion signals with signal to noise ratios above 2 were selected as precursors for MS/MS acquisition. During this data dependent analysis, an exclusion list with common trypsin autolysis masses and keratine masses was applied. External calibration in MS mode was performed using a mixture of five peptides: des-Arg1-Bradykinin ( $\mathrm{m} / \mathrm{z}=904.468)$, angioten$\sin I(m / z=1296.685)$, Glu1-fibrinopeptide $B(m / z=1570.677)$, and ACTH (18-39 clip) ( $\mathrm{m} / \mathrm{z}=2465.199)$, and ACTH (7-38 clip) $(\mathrm{m} / \mathrm{z}=3657.929)$. Similarly, tandem mass spectra were externally calibrated using known fragment ion masses observed in the MS/MS spectrum of Glu1-fibrinopeptide B.

Peaklists were created by using the "peaks to mascot" tool in the Explorer software of the ABSciex 5800 mass spectrometer. Common settings were signal to noise ratios of 2 and minimum peak areas of 10 . Data base searches were performed against an in-house created "Leishmania" (103645 sequences) database. The following search parameters were used: no restrictions on protein molecular weight, tryptic cleavage products including two tryptic missed cleavages allowed, variable modifications of cysteine (carbamidomethylation), methionine (oxidation), asparagine and glutamine (deamidation), and pyroglutamate formation at N-terminal glutamine of peptides. Decoy analysis revealed a false discovery rate of $0.8 \%$ considering peptide identity. It is worth mentioning that a second data base search against all entries (32611672 sequences) of the NCBI-non-redundant database (www.ncbi.nlm.nih.gov/index.html) revealed nearly the same results and did not suffer from losses in sensitivity of protein identification.

Gene Ontology (GO) biological process annotations for Leishmania proteins were assigned according to those reported in the Leishmania GeneDB database and confirmed using the Panther Classification System (http://www.pantherdb.org/ panther/globalSearch.jsp?).

\subsection{Western blotting}

Two-dimensional SDS-PAGE $12 \%$ (7 cm, pH 4-7) was used to fractionate $150 \mu \mathrm{g}$ of promastigote protein extract from miltefosine-sensitive and -resistant isolates. The proteins were transferred onto PVDF membranes (Hybond, Amershan, UK) in a trans-blot semidry transfer unit (GE Healthcare) by applying a current of $1.6 \mathrm{~mA} / \mathrm{cm}^{2}$ for $2 \mathrm{~h}$. The membranes were rinsed with PBS-Tween $0.1 \%$ and incubated with blocking buffer (5\% low-fat milk powder in PBS-Tween $0.1 \%$ ) at $4{ }^{\circ} \mathrm{C}$ overnight. Trans-blotted proteins were probed overnight with a rabbit polyclonal anti-EF-1 $\beta$ antibody (Cedarlane, Canada) and a mouse polyclonal anti- $\alpha$-tubulin (Sigma-Aldrich, USA) antibody at dilutions of 1:50 and 1:1000, respectively. After washing three times in PBS-Tween $0.5 \%$ for $5 \mathrm{~min}$, the membranes were incubated with horseradish peroxidase (HRP)-conjugated secondary antibodies that were used at a 1:2000 dilution. Specific binding was revealed with a Western blotting detection ECL system (Amersham, UK) and exposed to a CCD camera (Kodak Gel Logig 1500 imaging system, USA). The eEF-1 $\beta$ and tubulin (used as normalizer) signals were processed by ImageJ software (Wayne Rasband, NIH, USA, http://rsb.info.nih.gov/ij/) from 2-DE blotting. The signal intensities were measured in equal size delimitated areas and were expressed as arbitrary units.

\section{Results}

\subsection{In vitro susceptibility of $\mathbf{L}$. infantum chagasi clinical isolates to miltefosine}

The susceptibility of clinical isolates to miltefosine was evaluated in vitro by intracellular amastigote assay. The isolate from the cured patient had an $\mathrm{EC}_{50}$ of $3.9 \mu \mathrm{M}$ and was considered miltefosine-sensitive (S), whereas the isolate from the relapsed patient had an $\mathrm{EC}_{50}>15 \mu \mathrm{M}$, the highest concentration tested, and was considered drug resistant (R).

\subsection{Comparative proteomics between L. infantum chagasi isolates differing in vitro susceptibility to miltefosine}

To comparatively analyse the 2D-DIGE, a total of 3 large format 2DE gels were obtained. Two-paired samples ( $\mathrm{S}$ and $\mathrm{R}$ ) were labelled alternatively with Cy3 or Cy5 and were run in 3 gels along with a pooled standard, which was labelled with Cy2. The images were analysed by Decyder 2D software, v7.0. The image analysis showed that the average number of spots detected by CyDyes stains was $810 \pm 11.8$. Protein spots presented migration positions from 14 to $100 \mathrm{kDa}$ but were predominantly clustered between 14 and $70 \mathrm{kDa}$ (Fig. 1).

A differential analysis between drug-sensitive and -resistant isolates was performed on 823 matched spots. A protein spot was considered to have differential abundance if the spot was detected in all of the experimental replicates with statistical significance $(\alpha<0.05)$. In the image analysis, 46 protein spots showed significant differences when comparing the miltefosinesensitive and -resistant isolates. Out of the 46 spots, 26 (56.5\%) showed greater and 20 (43.5\%) showed lower expression of the resistant isolate compared to the sensitive isolate (Fig. 1). These differential spots were cut from the gels, digested, and used for MALDI-TOF/TOF analysis.

\subsection{Identification of spots with differential abundances by MS/MS}

With MALDI-TOF/TOF analysis, 41 (89.1\%) out of the 46 differentially regulated protein spots were successfully identified with high confidence after searching databases. From these spots were identified 50 proteins correspondent to 34 non-redundant proteins. The molecular mass and isoelectric point values estimated by gel electrophoresis (experimental) showed a good correlation with predicted values of the identified proteins $(r=0.97, p<0.0001$ for the molecular weight; and $r=0.69, p<0.0001$ for the isoelectric point). However, some variations were observed suggesting that fragments or post-translational protein modifications were visualised (Supplementary material S1). 
A
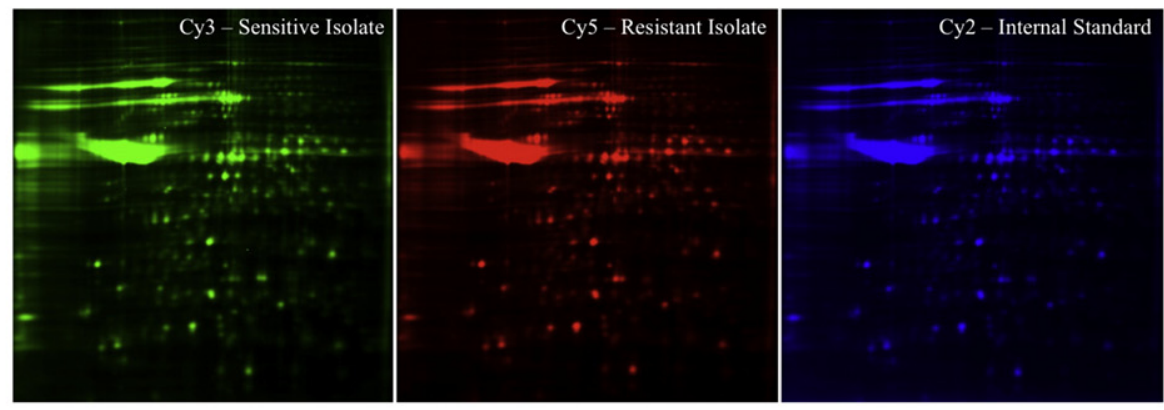

B

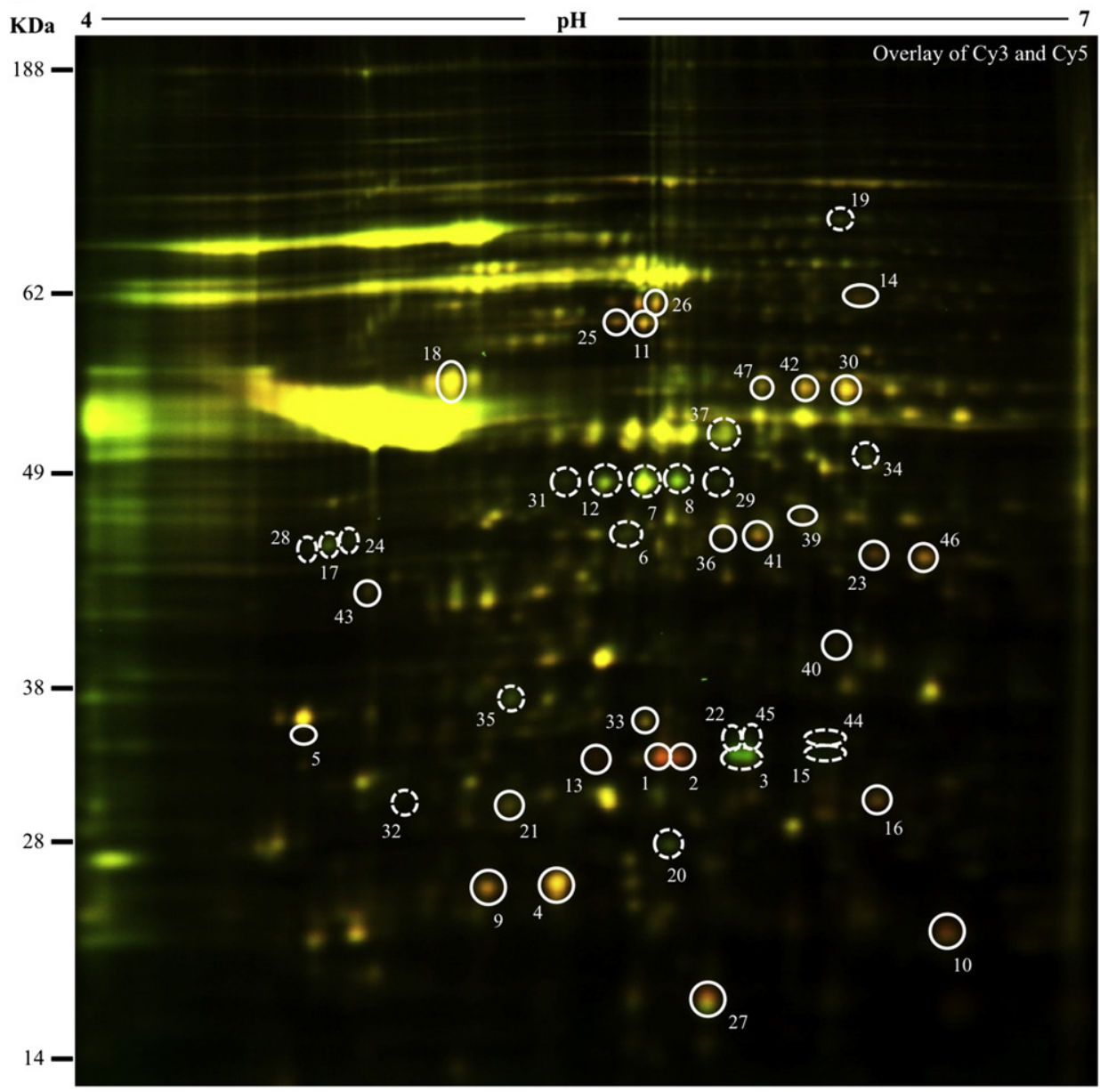

Fig. 1 - The 2D-DIGE comparative proteomics analysis between miltefosine-sensitive and -resistant L. infantum chagasi clinical isolates. (A) Representative 2D gels comprising L. infantum chagasi miltefosine-sensitive, miltefosine-resistant, and internal standard (mix of sensitive and resistant samples). (B) Overlay of Cy3 and Cy5 derived from a single gel, highlighting differentially expressed protein spots: solid line for over-regulation and a dashed line for down-regulation in the resistant isolate. The molecular weight markers (MW) are indicated. The highlighted spots were excised and identified by MALDI-TOF/ TOF. Information about the proteins corresponding to the spot numbers is listed in Tables 1 and 2 and in Supplementary material S2.

Nine spots were excluded upon further analysis, because they corresponded to multiple proteins (Supplementary material S2). Thirty-two protein spots remained with unique protein identification correspondent to 22 non-redundant proteins.

Five proteins (chaperonin Hsp60, peroxiredoxin, NADPdependent alcohol dehydrogenase, 2,4-dihydroxyhept- 2-ene-1,7-dioic acid aldolase and one hypothetical protein) were identified in different localisations on 2D-Gel spots, indicating potentially diverse protein forms, thus suggesting the occurrence of post-translational modification of mutation and of the presence of different protein species, as shown in Tables 1 and 2, and in Supplementary material S2. 
The identified proteins were classified according to the Gene Ontology/Panther Annotation of Biological Processes and categorised into 12 diverse functional groups (Fig. 2). Up-regulated proteins identified in the miltefosine-resistant isolate were found to be mainly involved in antioxidant/ detoxification, protein folding/chaperones, and stress processes. Conversely, proteins involved in amino acid metabolism and cytoskeleton were down-regulated in the miltefosine-resistant isolate. Proteins with unknown biological functions/hypothetical proteins represented $14.3 \%$ and
$27 \%$ of the proteins in the miltefosine-resistant and -sensitive isolates, respectively.

\subsection{Western blot}

The validation of our proteomic data was performed using immunoblotting. According to protein fold changes and the availability of validation reagents, the translation elongation factor 1-beta protein (EF-1 $\beta$ ) was selected for verification of protein-expression changes between miltefosine-resistant and

\section{Table 1 - Up-regulated proteins in the miltefosine-resistant phenotype identified in the proteomic study.}

\begin{tabular}{|c|c|c|c|c|c|c|c|c|}
\hline \multirow[t]{2}{*}{ ID $^{a}$} & \multirow[t]{2}{*}{ Protein identity } & \multirow[t]{2}{*}{$\begin{array}{l}\text { Ratio } \\
(\mathrm{S} / \mathrm{R})^{\mathrm{b}}\end{array}$} & \multirow[t]{2}{*}{$\begin{array}{l}\text { T-test } \\
\text { (p) }\end{array}$} & \multirow[t]{2}{*}{$\begin{array}{c}\text { Accession } \\
\text { number }^{c}\end{array}$} & \multirow[t]{2}{*}{$\begin{array}{c}\mathrm{pI}^{\mathrm{d}} \\
\text { (exp/pred) }\end{array}$} & \multirow[t]{2}{*}{$\begin{array}{c}\mathrm{M}_{\mathrm{r}}^{\mathrm{e}} \\
\text { (exp/pred) }\end{array}$} & \multicolumn{2}{|c|}{$\begin{array}{c}\text { Reported } \\
\text { association to } \\
\text { drug sensitive } \\
\text { phenotype }\end{array}$} \\
\hline & & & & & & & Reg & Ref \\
\hline \multicolumn{9}{|c|}{ Protein folding/chaperones and stress proteins ${ }^{f}$} \\
\hline 11 & Chaperonin Hsp60, mitochondrial precursor & 1.28 & 0.014 & gi 134074164 & $5.71 / 5.33$ & $60 / 59.32$ & ] & {$[23,29]$} \\
\hline 25 & Chaperonin Hsp60, mitochondrial precursor & 1.35 & 0.026 & gi 134074164 & $5.64 / 5.33$ & $60 / 59.32$ & & \\
\hline 26 & Chaperonin Hsp60, mitochondrial precursor & 1.34 & 0.026 & gi 134074164 & $5.75 / 5.33$ & $61 / 59.32$ & & \\
\hline 14 & Stress-induced protein sti1 & 1.23 & 0.016 & gi 134067520 & $6.33 / 5.90$ & $62 / 62.20$ & [/] & {$[24] /[29]$} \\
\hline \multicolumn{9}{|c|}{ Antioxidant/detoxification $^{\mathrm{f}}$} \\
\hline 4 & Mitochondrial peroxiredoxin & 1.36 & 0.007 & gi 16751316 & $5.47 / 6.43$ & $25 / 25.35$ & [/] & {$[21,29,49] /[21]$} \\
\hline 9 & Mitochondrial peroxiredoxin & 1.38 & 0.008 & gi 16751316 & $5.27 / 6.43$ & $24 / 25.35$ & & \\
\hline 10 & Cytosolic peroxiredoxin & 1.42 & 0.011 & gi 16751318 & $6.57 / 7.72$ & $21 / 22.12$ & & \\
\hline \multicolumn{9}{|c|}{ Protein biosynthesis ${ }^{\mathrm{f}}$} \\
\hline 5 & Translation elongation factor 1-beta & 1.11 & 0.007 & gi 134074101 & $4.74 / 4.61$ & $36 / 23.22$ & ] & [20] \\
\hline \multicolumn{9}{|c|}{ Protein targeting and signal transduction ${ }^{\mathrm{f}}$} \\
\hline 40 & Activated protein kinase c receptor (LACK) & 1.15 & 0.043 & gi 321398491 & $6.26 / 6.05$ & $40 / 34.35$ & ] & [50] \\
\hline \multicolumn{9}{|c|}{ DNA replication and repair ${ }^{\mathrm{f}}$} \\
\hline 43 & Proliferative cell nuclear antigen (PCNA) & 1.25 & 0.044 & gi 134068565 & $4.93 / 4.82$ & $43 / 32.39$ & प/[ & {$[29,47] /[51]$} \\
\hline \multicolumn{9}{|c|}{ Respiratory electron transport chain ${ }^{\mathrm{f}}$} \\
\hline 18 & ATPase beta subunit & 1.15 & 0.019 & gi 134070254 & $5.17 / 5.14$ & $55 / 56.26$ & [/] & {$[24,29] /[29]$} \\
\hline \multicolumn{9}{|c|}{ Metabolic enzymes ${ }^{\mathrm{f}}$} \\
\hline \multicolumn{9}{|c|}{ Carbohydrate metabolism } \\
\hline 23 & NADP-dependent alcohol dehydrogenase & 1,43 & 0.026 & gi 134069817 & $6.37 / 5.96$ & $44 / 38.43$ & - & - \\
\hline 46 & NADP-dependent alcohol dehydrogenase & 1.35 & 0.048 & gi 134069817 & $6.51 / 5.96$ & $44 / 38.43$ & - & - \\
\hline \multicolumn{9}{|c|}{ Amino acid metabolism } \\
\hline 1 & 2,4-Dihydroxyhept-2-ene-1,7-dioic acid aldolase & 1.85 & 0.007 & gi 134070342 & $5.76 / 5.80$ & $33 / 30.38$ & ] & {$[21,29]$} \\
\hline 2 & 2,4-Dihydroxyhept-2-ene-1,7-dioic acid aldolase & 1.78 & 0.007 & gi 134070342 & $5.81 / 5.80$ & $33 / 30.38$ & & \\
\hline 13 & 2,4-Dihydroxyhept-2-ene-1,7-dioic acid aldolase & 1.92 & 0.015 & gi 134070342 & $5.61 / 5.80$ & $33 / 30.38$ & & \\
\hline 16 & Pyrroline-5-carboxylate reductase & 1.25 & 0.016 & gi 321399182 & $6.38 / 6.22$ & $30 / 28.65$ & - & - \\
\hline \multicolumn{9}{|c|}{ Monosaccharide and phospholipid metabolic process/translation } \\
\hline 41 & GDP-mannose pyrophosphorylase & 1.24 & 0.043 & gi 134069789 & $6.04 / 5.70$ & $45 / 41.81$ & - & - \\
\hline \multicolumn{9}{|c|}{ Unknown biological process/hypothetical proteins ${ }^{\mathrm{f}}$} \\
\hline 27 & Ribonucleoprotein p18, mitochondrial precursor & 1.21 & 0.027 & gi 134068463 & $5.89 / 6.74$ & $17 / 21.30$ & ? & [52] \\
\hline 21 & Conserved hypothetical protein & 1.11 & 0.026 & gi 134073755 & $5.33 / 5.82$ & $30 / 29.11$ & - & - \\
\hline 36 & Conserved hypothetical protein & 1.28 & 0.036 & gi 134071523 & $5.95 / 6.11$ & $45 / 35.58$ & - & - \\
\hline
\end{tabular}

Reg - regulation, Ref - reference.

a Spot ID - The numbers correspond to the specific spots as indicated in Fig. 1.

b Average ratio DIGE (miltefosine-resistant (R) and miltefosine-resistant (S) phenotypes).

c Accession number corresponds to Leishmania infantum genome database according to NCBI.

d $\mathrm{pI}$ - Isoelectric point.

e $\mathrm{Mr}$ - Molecular weight $\mathrm{kDa}$ (exp - experimental and pred — predicted).

${ }^{\mathrm{f}}$ Functional categories according to Gene Ontology and Panther biological process annotations. 
Table 2 - Up-regulated proteins, in the miltefosine-sensitive phenotype, identified in the proteomic study.

\begin{tabular}{|c|c|c|c|c|c|c|c|c|}
\hline \multirow[t]{2}{*}{ ID $^{a}$} & \multirow[t]{2}{*}{ Protein identity } & \multirow[t]{2}{*}{$\begin{array}{l}\text { Ratio } \\
(\mathrm{S} / \mathrm{R})^{\mathrm{b}}\end{array}$} & \multirow[t]{2}{*}{$\begin{array}{l}\text { T-test } \\
\text { (p) }\end{array}$} & \multirow[t]{2}{*}{$\begin{array}{c}\text { Accession } \\
\text { number }^{c}\end{array}$} & \multirow[t]{2}{*}{$\begin{array}{c}\mathrm{pI}^{\mathrm{d}} \\
\text { (exp/pred) }\end{array}$} & \multirow[t]{2}{*}{$\begin{array}{c}\mathrm{M}_{\mathrm{r}}^{\mathrm{e}} \\
\text { (exp/pred) }\end{array}$} & \multicolumn{2}{|c|}{$\begin{array}{c}\text { Reported } \\
\text { association to drug } \\
\text { sensitive } \\
\text { phenotype }\end{array}$} \\
\hline & & & & & & & Reg & Ref \\
\hline \multicolumn{9}{|c|}{ Proteolysis $^{\mathrm{f}}$} \\
\hline 19 & Metallo-peptidase, Clan MA(E), Family M3 & 1.24 & 0.019 & gi 134070535 & $6.27 / 5.84$ & $68 / 77.18$ & ? & {$[20,29,47]$} \\
\hline \multicolumn{9}{|c|}{ Metabolic enzymes ${ }^{\mathrm{f}}$} \\
\hline \multicolumn{9}{|c|}{ Amino acid metabolism } \\
\hline 35 & Spermidine synthase (SPDSYN) & 1.41 & 0.036 & gi 134066976 & $5.34 / 5.18$ & $37 / 32.91$ & - & - \\
\hline 37 & S-adenosylmethionine synthetase & 1.20 & 0.036 & gi 321398550 & $5.95 / 5.50$ & $52 / 43.10$ & ] & {$[23,24,29,33]$} \\
\hline 44 & Nitrilase & 1.27 & 0.047 & gi 134070599 & $6.25 / 5.99$ & $34 / 31.11$ & ] & [29] \\
\hline 45 & 2,4-Dihydroxyhept-2-ene-1,7-dioic acid aldolase & 2.19 & 0.048 & gi 134070342 & $6.01 / 5.80$ & $34 / 30.38$ & ] & [21] \\
\hline \multicolumn{9}{|c|}{ Translation } \\
\hline 34 & RNA helicase & 1.23 & 0.035 & gi 134069547 & $6.34 / 8.82$ & $50 / 58.76$ & प/[ & {$[29,51] /[20]$} \\
\hline \multicolumn{9}{|c|}{ Cytoskeleton proteins ${ }^{f}$} \\
\hline 7 & Actin & 1.43 & 0.007 & gi 134067044 & $5.77 / 5.41$ & $52 / 42.02$ & [/] & {$[23] /[23,29,52]$} \\
\hline 12 & Actin & 1.36 & 0.014 & gi 134067044 & $5.60 / 5.41$ & $48 / 42.02$ & & \\
\hline \multicolumn{9}{|c|}{ Unknown biological process/hypothetical proteins ${ }^{f}$} \\
\hline 17 & Conserved hypothetical protein & 1.51 & 0.019 & gi 134066959 & $4.81 / 4.72$ & $45 / 39.14$ & - & - \\
\hline 24 & Conserved hypothetical protein & 1.51 & 0.026 & gi 134066959 & $4.88 / 4.72$ & $45 / 39.14$ & - & - \\
\hline 28 & Conserved hypothetical protein & 1.47 & 0.027 & gi 134066959 & $4.75 / 4.72$ & $45 / 39.14$ & - & - \\
\hline $\begin{array}{l}\text { Reg } \\
\text { a } \mathrm{Spc} \\
\text { b } \mathrm{Av} \\
\text { c } \mathrm{Acc} \\
\text { d } \mathrm{pI} \\
\text { e } \mathrm{Mr}\end{array}$ & $\begin{array}{l}\text { - regulation, Ref - reference. } \\
\text { ot ID - The numbers correspond to the specific sp } \\
\text { erage ratio DIGE (miltefosine-resistant (R) and milt } \\
\text { cession number corresponds to Leishmania infantum } \\
\text { - Isoelectric point. } \\
\text { - Molecular weight } \mathrm{kDa} \text { (exp - experimental and }\end{array}$ & $\begin{array}{l}\text { ts as ind } \\
\text { fosine-re } \\
\text { genome } \\
\text { pred - p }\end{array}$ & $\begin{array}{l}\text { ated in F } \\
\text { istant (S) } \\
\text { atabase a } \\
\text { edicted). }\end{array}$ & $\begin{array}{l}\text { ig. } 1 . \\
\text { phenotypes). } \\
\text { ccording to NC }\end{array}$ & BII. & & & \\
\hline
\end{tabular}

-sensitive isolates. Tubulin was used as a normaliser and this verification was carried out by 2D western blotting followed by densitometry analysis. Both proteins were well-detected and EF-1ß up-regulation in miltefosine-resistant phenotype was confirmed (Fig. 3). Densitometry analysis confirmed the visual evaluation of the immunoblotting signals, and the ratio of the signals between the strains (R/S) was 1.05 and 1.57 arbitrary units for $\alpha$-Tubulin and EF-1 $\beta$, respectively. These data indicate that

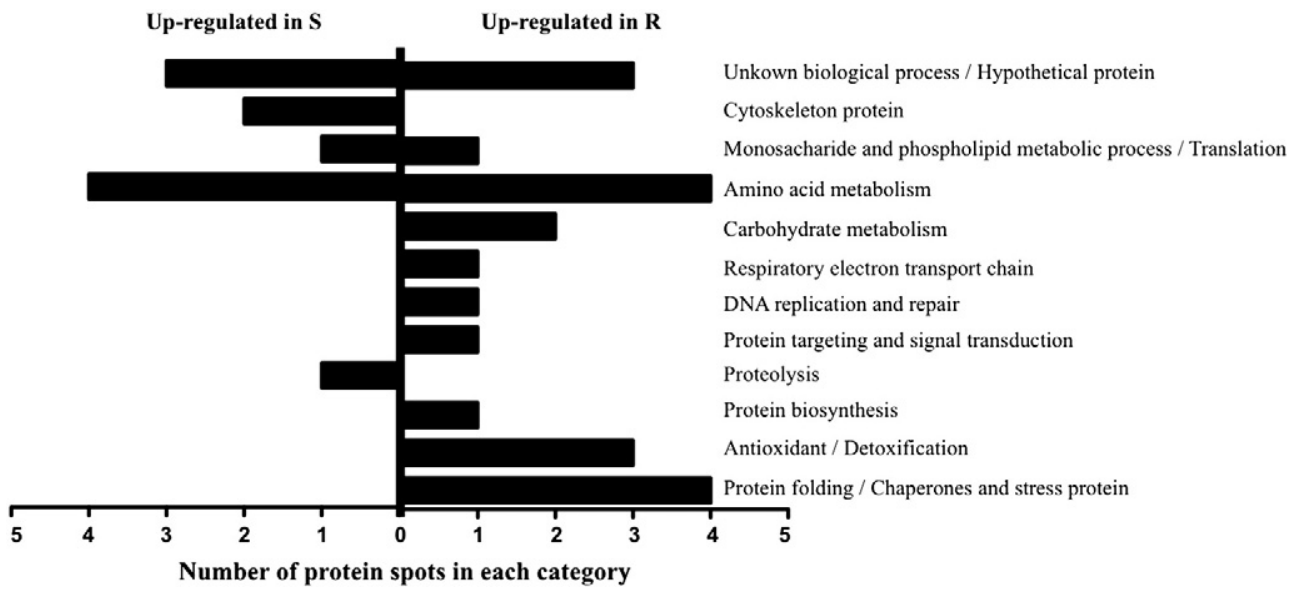

Fig. 2 - Functional assignment of identified proteins. Classes of biological process of up-regulated protein spot with unique protein identification in the miltefosine-resistant and -sensitive isolates. Protein functional classification was based on Gene Ontology and Panther annotations. Miltefosine-sensitive (S) and miltefosine-resistant (R) L. infantum chagasi clinical isolates. 
$\mathrm{EF}-1 \beta$ is up-regulated in the miltefosine-resistant isolate, corroborating the results from our proteomic analysis.

\section{Discussion}

Miltefosine has proven to be highly effective against visceral leishmaniasis in India [18,19]. However, around 50\% of VL patients treated with miltefosine relapse in Brazil. In addition, most isolates from relapsed patients were resistant in vitro to miltefosine, suggesting that treatment failure can be associated with natural resistance of the infecting parasite (Dietze R., unpublished data). In this scenario, proteomics screens have been useful in pinpointing novel drug resistance

A

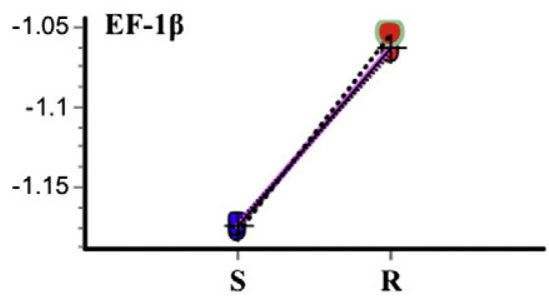

B

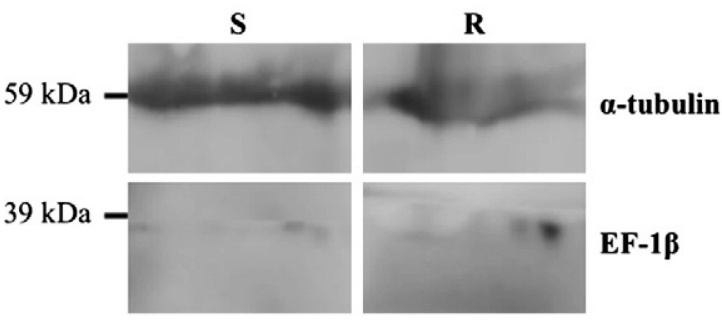

C

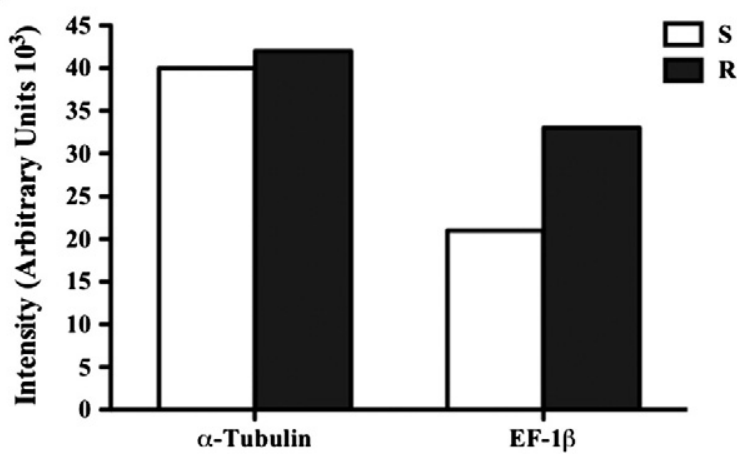

Fig. 3 - The validation of proteomic data. (A) Graphical representation of EF-1 $\beta$ protein spots up-regulated in miltefosine-resistant isolate $(p<0.01)$. Protein spot quantification was performed by the Differential In-gel Analysis module of the DeCyder 7.0 software. Each circle (blue or red) represents the abundance of the referred spot in an individual gel, expressed as a volume ratio to its corresponding internal standard. (B) The 2D western blot image of the independent verification of EF-1 $\beta$ and $\alpha$-tubulin (normaliser) proteins in miltefosine-sensitive (S) and -resistant (R) isolates. (C) Densitometry analysis of EF-1 $\beta$ and $\alpha$-tubulin (normaliser) proteins in miltefosine-sensitive (S) and -resistant $(R)$ isolates. mechanisms in Leishmania parasites [12,20-24]. Thus, a comparative proteomic approach was carried out and revealed significant changes in protein expression between isolates with different miltefosine susceptibilities.

In our study, soluble proteomes of miltefosine-sensitive and -resistant L. infantum chagasi were separated with a high-resolution 2D-DIGE, with a mean number of 810 spots. This number covered $10.1 \%$ of the $~ 8000$ open reading frames predicted for the Leishmania genome sequence, although post-translational modification and processing will significantly reduce this estimate. The total number of spots is slightly lower than that found in other studies of DIGE analysis on Leishmania infantum $[25,26]$ and much lower than that found by Daneshvar et al. [21]. Indeed, the total number of spots visualized in the DIGE can be influenced by several factors such as sample type (species/strains of parasite), sample preparation, size and the $\mathrm{pH}$ range of IPG strip, size of the gel, and also the detection parameters used. A total of 32 protein spots with unique protein identification (22 non-redundant) were differentially expressed between miltefosine-sensitive and -resistant L. infantum chagasi isolates, which were clearly grouped by ontology into 12 functional categories. Most are involved in redox regulation and stress response functions. Some of these proteins have already been associated with resistance to different drugs in Leishmania spp. (see references in Tables 1 and 2).

The functional categories with the highest number of differentially expressed spots in resistant isolate were chaperones and stress proteins. Chaperones, also termed heat shock proteins (Hsps), comprise several highly conserved families of protein folding facilitators that play important roles in many aspects of cell function, including responses to chemical and physiological stresses. These proteins have been associated with cell protection, preventing programmed cell death activation by modulating multiple events within apoptotic pathways (for a review, see $[27,28])$. In our analysis, three protein spots corresponding to the same chaperone Hsp60, were up-regulated in the miltefosine resistant isolate. These spots could represent different variants of Hsp60 with different post-translational modifications, because a small difference between the calculated and experimental pI of the identified spots was found. Over-regulation of this protein was observed in L. infantum chagasi and Leishmania braziliensis antimonial-resistant lines [29] and in L. major methotrexate-resistant lines [23]. In this study, one protein spot corresponded to stress-induced protein sti1 exhibited higher abundance in the resistant isolate. This protein forms a complex with heat shock proteins Hsp70 and Hsp90 (known as Hsp83 in Leishmania spp.) [30], acts as co-chaperone, and is produced in response to stress [31,32]. Considering this protein-protein interaction, the up-regulation of this stress protein could be associated with the resistant phenotype since the over-expression of Hsp83 has been linked to miltefosine and antimonial resistance in L. donovani [12]. Furthermore, Hsp83 interferes with the mitochondrial membrane potential, decreasing the drug-mediated programmed cell death in L. donovani [12], which has been described as an action mechanism of miltefosine $[13,33,34]$.

In addition, pronounced changes in the peroxirredoxin protein, which belong to the antioxidant/detoxification category, were observed in this proteomic study. This protein was 
identified in three different protein spots, represented by two mitochondrial and one cytosolic peroxirredoxin, and all spots showed up-regulation in the miltefosine-resistant isolate. Peroxirredoxin is found in different organisms and has been implicated in a wide variety of cell functions, such as redox homeostasis, apoptosis, signal transduction, and pathogen infection [35-38]. Peroxirredoxin is best known for its ability as an antioxidant enzyme that can use different reactive oxygen species (ROS) and reactive nitrogen species (RNS) as substrates. In the Trypanosomatidae family, peroxirredoxins are the major antioxidant enzymes [39]. Overexpression of this antioxidant protein has been associated previously with antimonial resistance in L. infantum chagasi and L. braziliensis [29] and with gentamicin resistance in L. infantum [21]. Harder et al. [40] showed that L. donovani parasites overexpressing peroxirredoxin are protected from hydrogen peroxide-induced programmed cell death. In accordance with these findings, a recent study indicated that miltefosine-unresponsive $L$. donovani has better redox machinery, which removes ROS more efficiently, improving its ability to maintain redox homeostasis [41].

In the current work, we highlighted other two proteins overexpressed in the resistant isolate, NADP-dependent alcohol

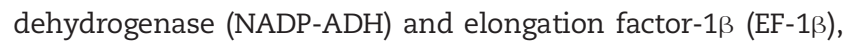
and although they are sorted into carbohydrate metabolism and protein biosynthesis, respectively, they also play an important role in redox homeostasis. NADP-ADH, known as NADP-aldehyde-reductase, comprises a class of oxidoreductases that catalyses the reversible oxidation of ethanol to acetaldehyde with the concomitant reduction of NADPH [42]. It has been shown that in yeast, during oxidative stress conditions (the glutathione levels are depleted and the glyoxalase pathway enzymes are inactive), aldose reductase activity is account for $40 \%$ of detoxification of the methylglyoxal in the cell [43]. EF-1 $\beta$ complex is a functionally distinct part of elongation factor 1. This complex is involved in ribosomal protein synthesis and simultaneously displays distinct activities, including those of trypanothione S-transferase and peroxidase $[44,45]$. The trypanothione-dependent system is common to all Leishmania species and plays an important role in parasite survival by neutralising the harmful effects of ROS (for a review, see [46]).

Although previous studies have reported the overexpression of enzymes that are involved in the trypanothione biosynthesis system in antimonial- and methotrexateresistant Leishmania lines [23,24], our results showed a down-regulation of two enzymes (Spermidine synthase and S-adenosylmethionine synthetase) that are involved in this pathway in the miltefosine-resistant isolate.

The other differentially expressed proteins with highly specific functions identified in this work that could be associated with the miltefosine resistant phenotype were proliferative cell nuclear antigen (PCNA) and mitochondrial ATPase $\beta$-subunit. PCNA participates in the process of replication and DNA repair. In agreement with our findings, PCNA was found to be up-regulated in antimonial-resistant L. donovani clinical isolates [47] and in an antimonial-induced resistant L. braziliensis line [29]. The mitochondrial ATPase $\beta$-subunit is the catalytic element of a multi-subunit hydrogen ion $\left(\mathrm{H}^{+}\right)$transporter that in yeast has a potential functional/regulatory relationship with an $A B C$ efflux pump that is involved in multi-drug resistance [48]. Considering that miltefosine-resistance in Leishmania has been associated with an overexpression of $A B C$ transporters related to a decrease in

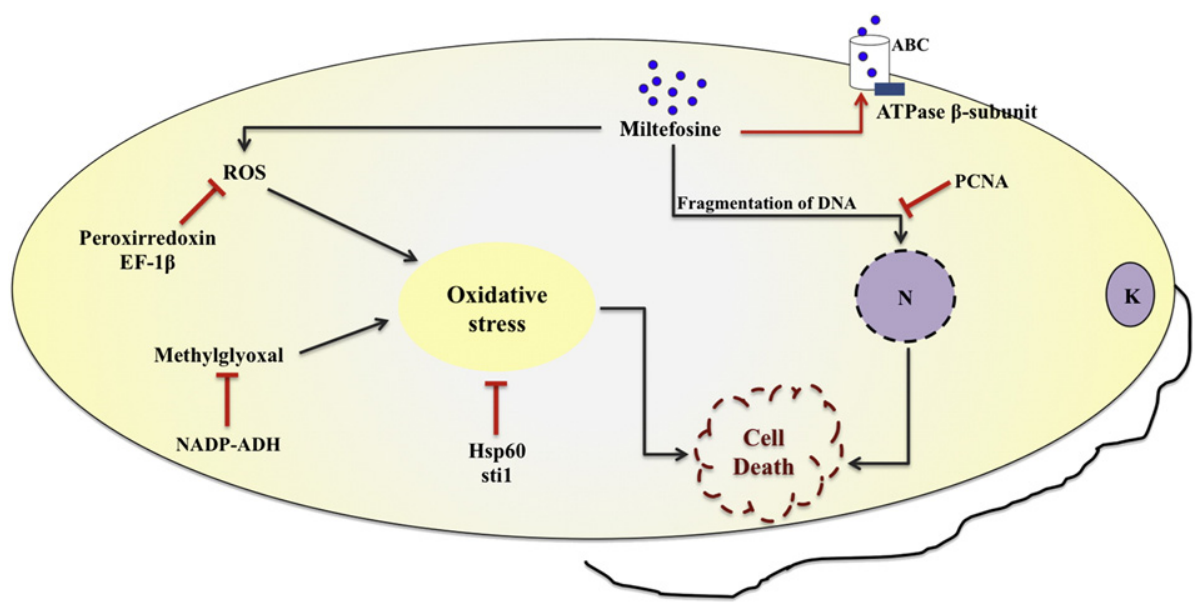

Fig. 4 - Proposed model for mechanism of miltefosine resistance in L. chagasi. The miltefosine (blue circles) induces the increased level of reactive oxygen species (ROS) which in turn leads to oxidative stress and then cell death [53]. The over expression of both Peroxirredoxin and elongation factor-1 $\beta$ (EF-1 $\beta$ ) proteins decrease the ROS level, providing protection against oxidative stress. In addition, the over expression of NADP-dependent alcohol dehydrogenase (NADP-ADH), chaperone Hsp60, and stress-induced protein sti1 (sti1) could also help to maintain redox homeostasis and then protect oxidative stress. Miltefosine is also able to induce cell death process by DNA fragmentation [13]. In this way, over expression of proliferative cell nuclear antigen (PCNA) could be involved in miltefosine resistance by repairing and replicating DNA. The over expression of ATPase $\beta$-subunit (considered a catalytic element of ABC efflux pump) also protects miltefosine-induced cell death by decreasing intracellular drug accumulation. $\mathrm{N}-$ nucleus and $\mathrm{K}-$ kinetoplast. 
intracellular drug accumulation [10], the overexpression of this protein subunit in resistant isolate could be correlated with resistant phenotype.

In conclusion, we present, for the first time, a proteomic 2D-DIGE/MS analysis to elucidate the changes in expression between miltefosine-sensitive and -resistant L. infantum chagasi isolates from VL patients with different miltefosine treatment outcomes. Our study revealed overexpressed proteins in miltefosine-resistant isolate that plays a role in redox homeostasis, stress response, protection to apoptosis, and drug translocation, consistent with the resistance phenotype. These differentially expressed proteins are likely involved in miltefosine natural resistance and suggest that the miltefosine resistance mechanism in Leishmania is multifactorial. Based on the results of the present study we propose a mechanism of miltefosine resistance in L. infantum chagasi which is depicted in Fig. 4. We believe that these findings can contribute to better the understanding of the miltefosineresistance phenotype. In addition, some of these highlighted proteins, after a better characterization, could be used as biomarkers of miltefosine-resistance in Leishmania, helping to establish more appropriate drug treatment.

Supplementary data to this article can be found online at http://dx.doi.org/10.1016/j.jprot.2014.05.010.

\section{Transparency document}

The Transparency document associated with this article can be found, in the online version.

\section{Acknowledgment}

We thank Dr. Fausto E. L. Pereira for his precious contributions to this work. The study was supported by Conselho Nacional de Desenvolvimento Científico e Tecnológico-CNPq (Grant 478080/2009 3) and Fundação de Amparo a Pesquisa do Espírito Santo-FAPES (Grant 54694280/2011), Brazil. EML is grateful for the CNPq research fellowship (PQ).

\section{R E F E R E N C E S}

[1] Pearson RD, Sousa AQ. Clinical spectrum of Leishmaniasis. Clin Infect Dis 1996;22:1-13.

[2] Engel J. Miltefosine, the story of a successful partnership: disease endemic country - TDR - pharmaceutical industry (Zentaris). TDR News 2002;68:5.

[3] Sundar S, Jha TK, Thakur CP, Engel J, Sindermann H, Fischer $\mathrm{C}$, et al. Oral miltefosine for Indian visceral leishmaniasis. $\mathrm{N}$ Engl J Med 2002;347:1739-46.

[4] Bhattacharya SK, Sinha PK, Sundar S, Thakur CP, Jha TK, Pandey K, et al. Phase 4 trial of miltefosine for the treatment of Indian visceral leishmaniasis. J Infect Dis 2007;196:591-8.

[5] Sundar S, Singh A, Rai M, Prajapati VK, Singh AK, Ostyn B, et al. Efficacy of miltefosine in the treatment of visceral leishmaniasis in India after a decade of use. Clin Infect Dis 2012;55:543-50.
[6] Perez-Victoria FJ, Castanys S, Gamarro F. Leishmania donovani resistance to miltefosine involves a defective inward translocation of the drug. Antimicrob Agents Chemother 2003;47:2397-403.

[7] Perez-Victoria FJ, Gamarro F, Ouellette M, Castanys S. Functional cloning of the miltefosine transporter. A novel P-type phospholipid translocase from Leishmania involved in drug resistance. J Biol Chem 2003;278:49965-71.

[8] Perez-Victoria FJ, Sanchez-Canete MP, Castanys S, Gamarro F. Phospholipid translocation and miltefosine potency require both $L$. donovani miltefosine transporter and the new protein LdRos3 in Leishmania parasites. J Biol Chem 2006;281:23766-75.

[9] Sanchez-Canete MP, Carvalho L, Perez-Victoria FJ, Gamarro F, Castanys S. Low plasma membrane expression of the miltefosine transport complex renders Leishmania braziliensis refractory to the drug. Antimicrob Agents Chemother 2009;53:1305-13.

[10] Perez-Victoria JM, Perez-Victoria FJ, Parodi-Talice A, Jimenez IA, Ravelo AG, Castanys S, et al. Alkyl-lysophospholipid resistance in multidrug-resistant Leishmania tropica and chemosensitization by a novel P-glycoprotein-like transporter modulator. Antimicrob Agents Chemother 2001;45:2468-74.

[11] Rakotomanga M, Saint-Pierre-Chazalet M, Loiseau PM. Alteration of fatty acid and sterol metabolism in miltefosine-resistant Leishmania donovani promastigotes and consequences for drug-membrane interactions. Antimicrob Agents Chemother 2005;49:2677-86.

[12] Vergnes B, Gourbal B, Girard I, Sundar S, Drummelsmith J, Ouellette M. A proteomics screen implicates HSP83 and a small kinetoplastid calpain-related protein in drug resistance in Leishmania donovani clinical field isolates by modulating drug-induced programmed cell death. Mol Cell Proteomics 2007;6:88-101.

[13] Paris C, Loiseau PM, Bories C, Breard J. Miltefosine induces apoptosis-like death in Leishmania donovani promastigotes. Antimicrob Agents Chemother 2004;48:852-9.

[14] Volpini AC, Passos VM, Oliveira GC, Romanha AJ. PCR-RFLP to identify Leishmania (Viannia) braziliensis and L. (Leishmania) amazonensis causing American cutaneous leishmaniasis. Acta Trop 2004;90:31-7.

[15] Sesana AM, Monti-Rocha R, Vinhas SA, Morais CG, Dietze R, Lemos EM. In vitro activity of amphotericin B cochleates against Leishmania chagasi. Mem Inst Oswaldo Cruz 2011;106:251-3.

[16] Neuhoff V, Arold N, Taube D, Ehrhardt W. Improved staining of proteins in polyacrylamide gels including isoelectric focusing gels with clear background at nanogram sensitivity using Coomassie Brilliant Blue G-250 and R-250. Electrophoresis 1988;9:255-62.

[17] Vergote D, Bouchut A, Sautiere PE, Roger E, Galinier R, Rognon A, et al. Characterisation of proteins differentially present in the plasma of Biomphalaria glabrata susceptible or resistant to Echinostoma caproni. Int J Parasitol 2005;35:215-24.

[18] Sundar S, Gupta LB, Makharia MK, Singh MK, Voss A, Rosenkaimer F, et al. Oral treatment of visceral leishmaniasis with miltefosine. Ann Trop Med Parasitol 1999;93:589-97.

[19] Sundar S, Rosenkaimer F, Makharia MK, Goyal AK, Mandal AK, Voss A, et al. Trial of oral miltefosine for visceral leishmaniasis. Lancet 1998;352:1821-3.

[20] Biyani N, Singh AK, Mandal S, Chawla B, Madhubala R. Differential expression of proteins in antimony-susceptible and -resistant isolates of Leishmania donovani. Mol Biochem Parasitol 2011;179:91-9.

[21] Daneshvar H, Wyllie S, Phillips S, Hagan P, Burchmore R. Comparative proteomics profiling of a gentamicin-attenuated Leishmania infantum cell line identifies key changes in parasite thiol-redox metabolism. J Proteomics 2012;75:1463-71.

[22] Drummelsmith J, Brochu V, Girard I, Messier N, Ouellette M. Proteome mapping of the protozoan parasite Leishmania and 
application to the study of drug targets and resistance mechanisms. Mol Cell Proteomics 2003;2:146-55.

[23] Drummelsmith J, Girard I, Trudel N, Ouellette M. Differential protein expression analysis of Leishmania major reveals novel roles for methionine adenosyltransferase and S-adenosylmethionine in methotrexate resistance. J Biol Chem 2004;279:33273-80.

[24] Walker J, Gongora R, Vasquez JJ, Drummelsmith J, Burchmore $\mathrm{R}$, Roy $\mathrm{G}$, et al. Discovery of factors linked to antimony resistance in Leishmania panamensis through differential proteome analysis. Mol Biochem Parasitol 2012;183:166-76.

[25] Costa MM, Andrade HM, Bartholomeu DC, Freitas LM, Pires SF, Chapeaurouge AD, et al. Analysis of Leishmania chagasi by 2-D difference gel electrophoresis (2-D DIGE) and immunoproteomic: identification of novel candidate antigens for diagnostic tests and vaccine. J Proteome Res 2011;10:2172-84.

[26] da Fonseca Pires S, Fialho Jr LC, Silva SO, Melo MN, de Souza CC, Tafuri WL, et al. Identification of virulence factors in Leishmania infantum strains by a proteomic approach. J Proteome Res 2014;13:1860-72.

[27] Beere HM. "The stress of dying": the role of heat shock proteins in the regulation of apoptosis. J Cell Sci 2004;117:2641-51.

[28] Folgueira C, Requena JM. A postgenomic view of the heat shock proteins in kinetoplastids. FEMS Microbiol Rev 2007;31:359-77.

[29] Matrangolo FS, Liarte DB, Andrade LC, de Melo MF, Andrade JM, Ferreira RF, et al. Comparative proteomic analysis of antimony-resistant and -susceptible Leishmania braziliensis and Leishmania infantum chagasi lines. Mol Biochem Parasitol 2013;190:63-75.

[30] Hubel A, Clos J. The genomic organization of the HSP83 gene locus is conserved in three Leishmania species. Exp Parasitol 1996;82:225-8.

[31] Hombach A, Ommen G, Chrobak M, Clos J. The Hsp90-Sti1 interaction is critical for Leishmania donovani proliferation in both life cycle stages. Cell Microbiol 2012;15(4):585-600.

[32] Lassle M, Blatch GL, Kundra V, Takatori T, Zetter BR. Stress-inducible, murine protein mSTI1. Characterization of binding domains for heat shock proteins and in vitro phosphorylation by different kinases. J Biol Chem 1997;272:1876-84.

[33] Verma NK, Singh G, Dey CS. Miltefosine induces apoptosis in arsenite-resistant Leishmania donovani promastigotes through mitochondrial dysfunction. Exp Parasitol 2007;116:1-13.

[34] Verma NK, Dey CS. Possible mechanism of miltefosine-mediated death of Leishmania donovani. Antimicrob Agents Chemother 2004;48:3010-5.

[35] Nathan C, Shiloh MU. Reactive oxygen and nitrogen intermediates in the relationship between mammalian hosts and microbial pathogens. Proc Natl Acad Sci U S A 2000;97:8841-8.

[36] Droge W. The plasma redox state and ageing. Ageing Res Rev 2002;1:257-78.

[37] Wood ZA, Poole LB, Karplus PA. Peroxiredoxin evolution and the regulation of hydrogen peroxide signaling. Science 2003;300:650-3.
[38] Hofmann B, Hecht HJ, Flohe L. Peroxiredoxins. Biol Chem 2002;383:347-64.

[39] Wilkinson SR, Temperton NJ, Mondragon A, Kelly JM. Distinct mitochondrial and cytosolic enzymes mediate trypanothione-dependent peroxide metabolism in Trypanosoma cruzi. J Biol Chem 2000;275:8220-5.

[40] Harder S, Bente M, Isermann K, Bruchhaus I. Expression of a mitochondrial peroxiredoxin prevents programmed cell death in Leishmania donovani. Eukaryot Cell 2006;5:861-70.

[41] Das M, Saudagar P, Sundar S, Dubey VK. Miltefosine unresponsive Leishmania donovani has better ability of resist reactive oxygen species. FEBS J 2013;280(19):4807-15.

[42] Reid MF, Fewson CA. Molecular characterization of microbial alcohol dehydrogenases. Crit Rev Microbiol 1994;20:13-56.

[43] Gomes RA, Sousa Silva M, Vicente Miranda H, Ferreira AE, Cordeiro CA, Freire AP. Protein glycation in Saccharomyces cerevisiae. Argpyrimidine formation and methylglyoxal catabolism. FEBS J 2005;272:4521-31.

[44] Vickers TJ, Wyllie S, Fairlamb AH. Leishmania major elongation factor 1B complex has trypanothione S-transferase and peroxidase activity. J Biol Chem 2004;279:49003-9.

[45] Vickers TJ, Greig N, Fairlamb AH. A trypanothione-dependent glyoxalase I with a prokaryotic ancestry in Leishmania major. Proc Natl Acad Sci U S A 2004;101:13186-91.

[46] Colotti G, Ilari A. Polyamine metabolism in Leishmania: from arginine to trypanothione. Amino Acids 2011;40:269-85.

[47] Kumar A, Sisodia B, Misra P, Sundar S, Shasany AK, Dube A. Proteome mapping of overexpressed membrane-enriched and cytosolic proteins in sodium antimony gluconate (SAG) resistant clinical isolate of Leishmania donovani. Br J Clin Pharmacol 2010;70:609-17.

[48] Zhang X, Moye-Rowley WS. Saccharomyces cerevisiae multidrug resistance gene expression inversely correlates with the status of the $F(0)$ component of the mitochondrial ATPase. J Biol Chem 2001;276:47844-52.

[49] Wyllie S, Vickers TJ, Fairlamb AH. Roles of trypanothione S-transferase and tryparedoxin peroxidase in resistance to antimonials. Antimicrob Agents Chemother 2008;52:1359-65.

[50] El Fadili K, Drummelsmith J, Roy G, Jardim A, Ouellette M. Down regulation of KMP-11 in Leishmania infantum axenic antimony resistant amastigotes as revealed by a proteomic screen. Exp Parasitol 2009;123:51-7.

[51] Chawla B, Jhingran A, Panigrahi A, Stuart KD, Madhubala R. Paromomycin affects translation and vesicle-mediated trafficking as revealed by proteomics of paromomycin -susceptible -resistant Leishmania donovani. PLoS One 2011;6: e26660.

[52] Sharma S, Singh G, Chavan HD, Dey CS. Proteomic analysis of wild type and arsenite-resistant Leishmania donovani. Exp Parasitol 2009;123:369-76.

[53] Moreira W, Leprohon P, Ouellette M. Tolerance to drug-induced cell death favours the acquisition of multidrug resistance in Leishmania. Cell Death Dis 2011;2: e201. 